\title{
Emergence of polymyxin B-heteroresistant hypervirulent Klebsiella pneumoniae from an individual in the community with asymptomatic bacteriuria
}

\author{
Jun $\mathrm{Li}^{1,2}$, Mengli Tang ${ }^{1}$, Fengjun Xia ${ }^{1}$, Changhang Min ${ }^{1}$, Yongmei $\mathrm{Hu}^{1,2}$, Haichen Wang ${ }^{1,2}$ and Mingxiang Zou ${ }^{1,2^{*}}$
}

\begin{abstract}
Background: The heteroresistance of polymyxin B, a last-resort antibiotic used to treat many serious bacterial infections, may lead to antibiotic treatment failure. However, polymyxin B-heteroresistant isolates are rare in individuals living in the community. We report a polymyxin B-heteroresistant hypervirulent Klebsiella pneumoniae (hvKP) isolate from an individual in the community with asymptomatic bacteriuria.

Results: The NYTJ35 isolate had multiple virulence genes that encoded a mucoid phenotype regulator ( $r m p A)$, aerobactin (iuCABCD-iutA), salmochelin (iroBCDN), yersiniabactin (irp1-2 and ybtAEPQSTUX), and a truncated rmpA2. Infection of galleria mellonella larvae indicated the isolate was hypervirulent. Antimicrobial susceptibility testing showed it was susceptible to all tested antibiotics except polymyxin B. The proportion of surviving bacteria was $1.2 \times 10^{-7}$ based on the population analysis profile (PAP) method, suggesting the presence of polymyxin B heteroresistance. The isolate was not hypermucoviscous, but it was a strong biofilm producer. It had capsular serotype K1 and belonged to sequence type 23 (ST23). The isolate also had the D150G substitution in phoQ, which is known to confer polymyxin B resistance.
\end{abstract}

Conclusions: We identified the co-occurrence of hypervirulence and polymyxin B heteroresistance in a K. pneumoniae isolate from an individual with asymptomatic bacteriuria. We suggest the use of increased screening for hvKP in individuals living in the community.

Keywords: Hypervirulent K. pneumoniae, Polymyxin B heteroresistance, Asymptomatic bacteriuria, Communitydwelling adult, Urine specimen, Whole genome sequencing

\section{Background}

Klebsiella pneumoniae (KP) is one of the six ESKAPE pathogens (Enterococcus faecium, Staphylococcus aureus, Klebsiella pneumoniae, Acinetobacter baumannii, Pseudomonas aeruginosa, and Enterobacter spp.) that are leading causes of nosocomial infections throughout

\footnotetext{
*Correspondence: zoumingxiang@csu.edu.cn

1 Department of Clinical Laboratory, Xiangya Hospital, Central South

University, Changsha 410008, Hunan, China

Full list of author information is available at the end of the article
}

the world [1]. Hypervirulent K. pneumoniae (hvKP) has increased virulence and transmissibility, and is associated with a higher mortality rate [2]. The first report of hvKP was in Taiwan during 1986 [3], and subsequent studies identified hvKP in many other regions, including France, mainland China, Japan, Germany, the United States, Brazil, and Mexico [4-11]. This variant can infect the liver, lungs, and urinary tract. Hosoda et al. recently described a patient from Japan who had COVID-19 and a respiratory infection by hvKP and subsequently died 
from respiratory failure [7]. Therefore, hvKP is a pathogen that poses a great threat to human health [12].

Earlier studies reported that hvKP was susceptible to all antimicrobials with the exception of ampicillin, to which K. pneumoniae has intrinsic resistance. However, more recent studies reported the emergence of drug-resistant hvKP strains, such as those that produce extended spectrum beta-lactamase (ESBL) and others that have carbapenem resistance [4-6]. Until recently, polymyxin B was regarded as the last-resort antibiotic for the treatment of serious infections by multidrug resistant (MDR) Gram-negative bacteria. Unfortunately, several recent reports described nosocomial infections by polymyxin B-resistant hvKP $[13,14]$. The presence of polymyxin $B$ resistance is a significant challenge due to the limited availability of alterative effective antimicrobials. The cooccurrence of hypervirulence and MDR in K. pneumoniae is therefore a significant clinical challenge.

Heterogeneous antibiotic resistance may be considered a stage in the progression to antibiotic resistance [15]. There is currently only limited knowledge of polymyxin B-heteroresistance in hvKP, and the specific molecular and epidemiological characteristics of these infections are still unknown [16]. Asymptomatic bacteriuria (ASB), an common type of urinary tract infection (UTI), is defined by a positive urine culture without signs and symptoms in the patient. ASB mainly occurs in community-dwelling individuals infection [17]. ASB is more in the elderly, in that the prevalence is less than $2 \%$ for children and up to $50 \%$ in elderly residents of long-term care facilities [17]. The present study describes the isolation of a polymyxin B-heteroresistant hvKP strain from the urine sample of an asymptomatic male individual living in the community, and the molecular epidemiological characteristics of this strain, as a basis for preventing transmission.

\section{Results}

\section{Characteristics and identification of the hvKP isolate}

During routine mass screening for kidney disease, we found a $K$. pneumoniae isolate (NYTJ35) in the urine sample of an asymptomatic male individual who was 34 years-old and living in the community. This individual had no symptoms of UTI, and therefore received no treatment. However, his urine tested positive for white blood cells, protein, and nitrite. After collection and culturing of the uncontaminated urine, the colony count was $1 \times 10^{5}$ colony forming units $(\mathrm{CFU}) / \mathrm{mL}$.

\section{Antimicrobial susceptibility and gene resistance testing}

The antimicrobial susceptibility tests (Table 1) showed that NYTJ35 was sensitive to all tested antibiotics, including ceftazidime (CAZ), cefepime (FEP), imipenem
Table 1 Minimum inhibitory concentrations of different antibiotics for the NYTJ35 isolate ${ }^{a}$

\begin{tabular}{ll}
\hline Antibiotic & MIC $(\boldsymbol{\mu g} / \mathbf{m L})$ \\
\hline Ceftazidime & $\leq 1$ \\
Cefepime & $\leq 1$ \\
Imipenem & $\leq 0.5$ \\
Meropenem & $\leq 0.25$ \\
Ceftazidime/avibactam & $\leq 1$ \\
Aztreonam & $\leq 1$ \\
Piperacillin/tazobactam & 16 \\
Nitrofurantoin & $\leq 32$ \\
Amikacin & $\leq 4$ \\
Levofloxacin & 0.25 \\
Tigecycline & 1 \\
\hline
\end{tabular}

${ }^{\mathrm{a}}$ Determined using broth microdilution

(IPM), meropenem (MEM), ceftazidime/avibactam (CZA), aztreonam (ATM), piperacillin/tazobactam (TZP), nitrofurantoin (NIT), amikacin (AMK), levofloxacin (LVX), and tigecycline (TGC), but not polymyxin $B$ $(2 \mu \mathrm{g} / \mathrm{mL})$. We used the population analysis profile (PAP) method, the gold standard for detection of heteroresistance, to detect polymyxin B heteroresistance in NYTJ35. Thus, when the polymyxin B concentration was $8 \mu \mathrm{g} /$ $\mathrm{mL}$ (4-fold above its breakpoint of $2 \mu \mathrm{g} / \mathrm{mL}$ ), the number of colonies in the stock solution was $37 \mathrm{CFU}$; a control without polymyxin B led to $301 \mathrm{CFU}$ at a dilution of $10^{-6}$. As described in the Methods, this indicated that the proportion of surviving bacteria was $1.2 \times 10^{-7}$ (between $10^{-7}$ and $50 \%$ ) and that this strain was heteroresistant to polymyxin $\mathrm{B}$.

The NYTJ35 strain had the D150G substitution in phoQ, but had no mutations in the chromosomal genes $m g r B$, phoP, $p m r A$, or $p m r B$. We also did not detect the plasmid mediated $m c r$-like gene, suggesting this mutation may be responsible for the heteroresistance to polymyxin $B$.

\section{Virulence factors}

When grown on agar plates, the colony morphology of NYTJ35 was not mucoid and only formed strings less than $5 \mathrm{~mm}$, indicating it was not hypermucoviscous. The strain had a $\mathrm{K} 1$ capsule and multiple virulence genes encoding a mucoid phenotype regulator $(r m p A)$, aerobactin (iucABCD-iutA), salmochelin (iroBCDN), and yersiniabactin (irp1-2 and ybtAEPQSTUX). Notably, the isolate carried a truncated $r m p A 2$ gene.

\section{Biofilm formation}

Crystal violet staining indicated that NYTJ35 was a strong biofilm producer. (OD $>4 \times \mathrm{ODc}$ ). 


\section{Galleria mellonella infection model}

Analysis of the virulence of NYTJ35 indicated that the survival of G. mellonella larvae was $40.0 \%$, higher than from infection by ATCC 700603 and lower than from infection by NTUH-K2044. These results thus indicated that this strain was hypervirulent (Fig. 1).

\section{Phylogenic analysis}

We deposited the sequence data in the NCBI (PRJNA753708). The multi-locus sequence typing (MLST) result showed it belonged to ST23 (2-1-1-1-94-12). We performed phylogenetic analysis of NYTJ35 using BacWGSTdb 2.0 (SNP threshold: 500, MLST scheme with cgMLST, MLST threshold: 200). Based on the cgMLST strategy, we identified 33 close isolates, with 32 isolates in ST23 and 1 isolate in ST57. The most closely related isolate was ST23 FLVM01 (GenBank: FLVM00000000.1), with 108 different alleles. FLVM01 was from a human urine sample collected in Thailand on 13 June 2016 (Fig. 2).

\section{Discussion}

hvKP is highly pathogenic and transmissible, and the control of hvKP infections is challenging due to the limited availability of effective anti-infective therapies. Moreover, there is increasing drug resistance in hvKP. In particular, ESBL-producing strains have emerged, there is evidence of carbapenem resistance in nosocomial hvKP infections, and polymyxin B-resistant hvKP was recently identified. In particular, $\mathrm{Gu}$ et al. [18] detected polymyxin B-resistant hvKP in an infant with diarrhea and Huang et al. [19] reported polymyxin B-resistant hvKP in clinical specimens. Polymyxin B-heteroresistance may be considered a stage during the progression to antibiotic resistance, and patients with these infections usually experience treatment failure. Previous studies also detected polymyxin
B-heteroresistant isolates in classical $K$. pneumoniae (cKP) $[20,21]$, but few studies examined polymyxin B-heteroresistance in hvKP [16], especially from individuals in the community. Thus, the prevalence of polymyxin B-heteroresistance in hvKP in the community may be underestimated [22]. We isolated a polymyxin B-heteroresistant hvKP strain (NYTJ35) from the urine sample of a male individual in the community who had ASB, and analyzed its molecular and epidemiological characteristics to provide a basis for preventing transmission.

There is currently no agreement on the definition of hvKP. Initially, hypermucoviscosity (defined by a positive string test) was considered a critical characteristic of hvKP [23]. However, some studies found that certain hvKP strains did not have the hypermucoviscous phenotype [24]. Recent studies showed that multiple biomarkers that were common on the hvKP virulence plasmid, such as ${ }_{p} r m p A,{ }_{p} r m p A 2$, iucA, iroB, and peg-344, provided highly accurate identification of hvKP $[25,26]$. The present study examined virulence-related genes, hypermucoviscosity, capsular serotype, and virulence of the strain using the G. mellonella larva test.

We found that NYTJ35 was heteroresistant to polymyxin B, but was susceptible to other antimicrobial agents, in accordance with a report by Lu et al. [13] that described hypervirulent $K$. variicola. The most common mechanism of polymyxin $B$ resistance is chromosomal mutation in genes such as $p m r A, p m r B$, phoP, phoQ, and $m g r B$. The D150G substitution in $p h o Q$, one of the most common chromosomal mutations responsible for polymyxin B resistance [14], was also present in NYTJ35. Cheong et al. reported colistin heteroresistance in $K$. pneumoniae isolates due to diverse mutations of $P m r A B$ and $P h o P Q$ in resistant subpopulations, although they did not detect other chromosomal gene mutations in their isolates [27]. The $m c r$ plasmid gene is a transferable

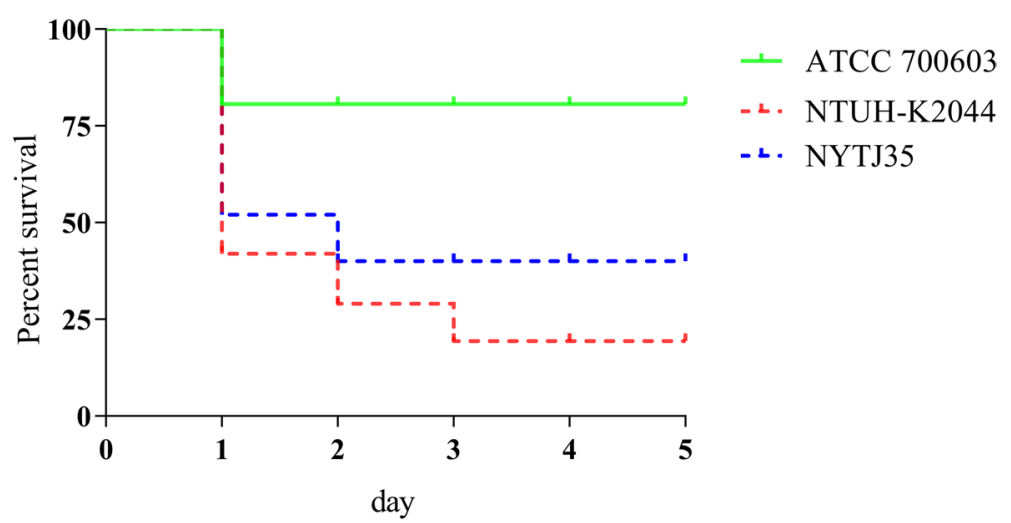

Fig. 1 Virulence of the NYTJ35 isolate. The G. mellonella infection model was used to determine the virulence of ATCC 700603 (low-virulence KP control), NTUH-K2044 (high-virulence KP control), and the NYTJ35 isolate 


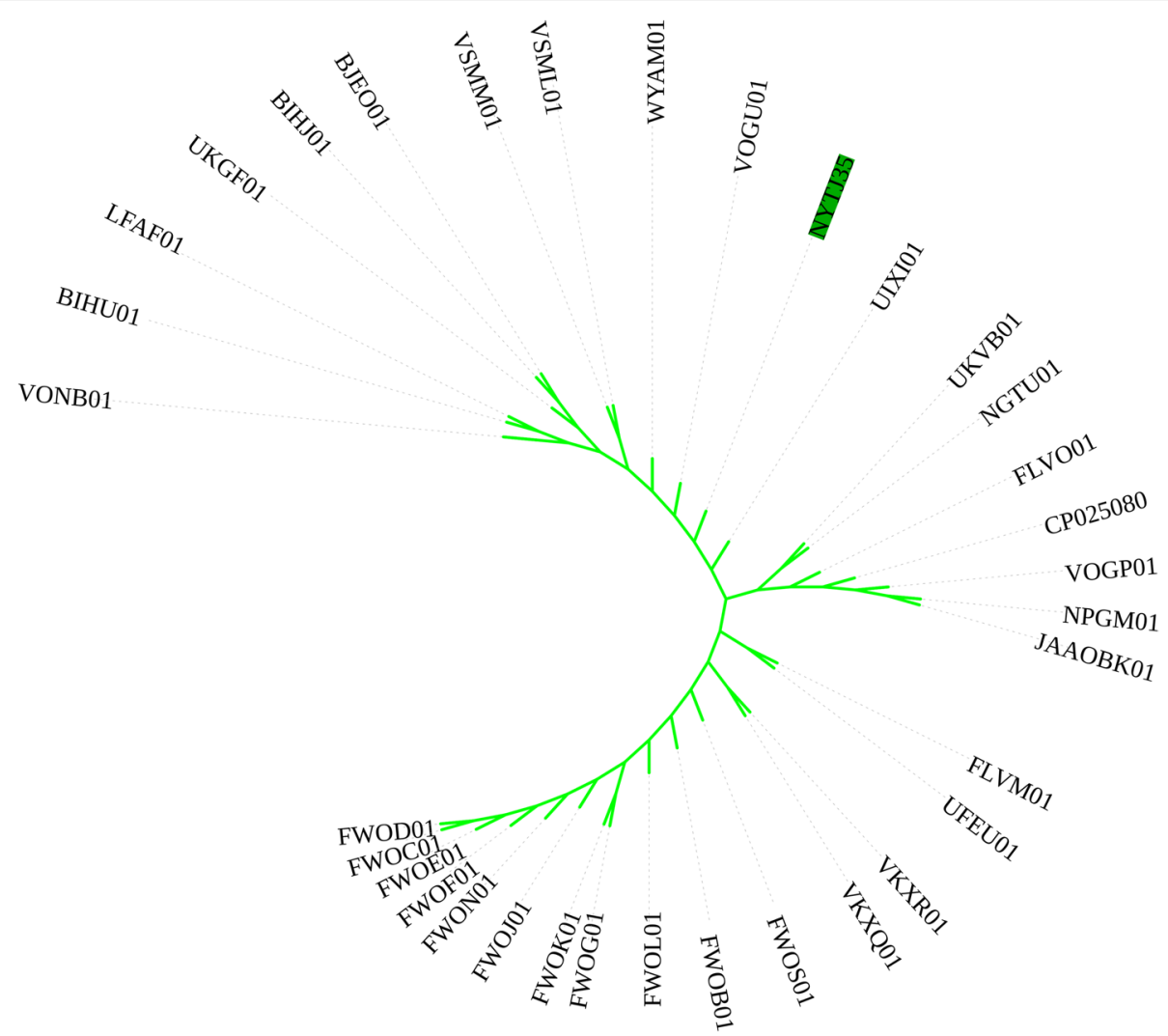

Fig. 2 Phylogenetic analysis of NYTJ35. Phylogenic analysis was performed using BacWGSTdb 2.0 with a single nucleotide polymorphism (SNP) threshold of 500, the MLST scheme with cgMLST, and a MLST threshold of 200. The cgMLST strategy led to the identification of 33 isolates that were in two STs (ST23: $n=32 ;$ ST57: $n=1$ ). NYTJ35 was most closely related to ST23 FLVM01 (GenBank: FLVM00000000.1), with 108 different alleles

polymyxin B resistance gene first discovered in 2015. Although this gene confers drug resistance transfer between humans and animals [28], it was not present in NYTJ35.

The hypermucoviscosity phenotype is an important feature of hvKP, but not all hvKP isolates have hypermucoviscosity [24]. NYTJ35 has genes encoding the mucoid phenotype regulator $(r m p A)$, but had a truncated $r m p A 2$ that presumably led to the non-hypermucoviscous phenotype, suggesting that the loss of hypermucoviscosity in polymyxin B-heteroresistant hvKP may increase its fitness [29]. In particular, the loss of hypermucoviscosity may reduce adhesion of these bacteria to human cells, such as neutrophils [30]. Notably, although NYTJ35 was not hypermucoviscous, it was a strong biofilm producer. This may be related to its ability to escape immune cells in the urinary tract and thus increase colonization [31].

There are multiple serotypes of hvKP, but most isolates are $\mathrm{K} 1$ or $\mathrm{K} 2$ [32]. Previous studies showed that isolates with the K1 serotype were mainly in ST23, whereas isolates with the $\mathrm{K} 2$ serotype were in many
STs, including ST25, ST65, ST66, and ST86 [33-36]. We found that NYTJ35 had the K1 serotype and was in ST23, an ST common in hospital-acquired and community-acquired infections. We also found that this isolate's closest known relative was FLVM01 (GenBank: FLVM00000000.1), although they had 108 different alleles, suggesting they may only be distantly related.

\section{Conclusion}

To our knowledge, this study is the first to detect a polymyxin B-heteroresistant hvKP isolate in a urine specimen of an individual from the community with asymptomatic bacteriuria. The polymyxin B-heteroresistant strain described here possibly had increased fitness due to its loss of hypermucoviscosity, which could impede its adhesion to immune cells. The co-occurrence of hypervirulence and polymyxin $B$ heteroresistance in a K. pneumoniae isolate from an asymptomatic individual living in the community suggests the need for increased surveillance. 


\section{Methods}

\section{Bacterial strain}

On 20 November 2020, NYTJ35 was isolated at Xiangya Hospital of Central South University (Changsha, China) from the urine sample of a healthy male individual who was living in the community. This isolate was identified using matrix-assisted laser desorption/ionization timeof-flight mass spectrometry (MALDI-TOF MS; Bruker Daltonics GmbH, Bremen, Germany). Briefly, one colony from an overnight culture was taken with a disposable loop and spotted onto a metal plate and the spots were then covered with $1 \mu \mathrm{L}$ of a $\alpha$-cyano-4-hydroxycinnamic acid (HCCA) matrix (Bruker Daltonik $\mathrm{GmbH}$, Bremen, Germany). Then, bacterial samples on the microplate were analyzed with MALDI-TOF MS. Finally, MALDI Biotyper ${ }^{\circledR}$ (Bruker Daltonik GmbH, Bremen, Germany) software was used to classify the isolate at the genus and species level. The quality control strain was Escherichia coli ATCC 25922 (National Center for Clinical Laboratories, Beijing, China).

\section{Antimicrobial susceptibility testing}

The broth microdilution method was used to determine the minimum inhibitory concentrations (MICs) of the following antimicrobial agents: CAZ, FEP, IPM, MEM, CZA, ATM, TZP, NIT, AMK, LVX, TGC, and polymyxin B. All results were interpreted according to Clinical and Laboratory Standards Institute (CLSI) [37]. The MIC of TGC was reported in accordance with the breakpoint established by the U.S. Food and Drug Administration. E. coli ATCC 25922 was used for quality control.

Polymyxin B-heteroresistance in hvKP was determined by the PAP method, the gold standard for detecting heteroresistance [38]. Briefly, this technique quantifies the proportion of resistant cells within a culture in response to different antibiotic concentrations. Heteroresistance was considered present when the proportion of surviving bacteria at a polymixin B concentration at least 4-fold above its breakpoint was between $10^{-7}$ and $50 \%$. This was calculated as: (number of colonies on polymyxin $B$ plate $\times$ dilution factor) $/$ (number of colonies on antibiotic free plate $\times$ dilution factor $)$.

\section{Detection of capsular serotypes, mucoviscous phenotype, and biofilm formation}

After overnight culturing, the genomic DNA of NYTJ35 was extracted using a boiling method [39]. The polymerase chain reaction (PCR) was used to detect capsular serotype genes (K1, K2, K5, K20, K54, K57) using primers as previously described [2]. Positive PCR products were then subjected to direct Sanger sequencing.
The mucoviscous phenotype was evaluated using the string test [40]. The isolate was cultured overnight on a blood agar plate at $37^{\circ} \mathrm{C}$, and a bacterial colony was then stretched with an inoculation loop. Hypermucoviscosity was defined by the formation of viscous strings that were at least $5 \mathrm{~mm}$ in length.

Crystal violet staining was used to test biofilm formation by NYTJ35 [41]. Absorbance was measured at $570 \mathrm{~nm}$, and data are presented as the means \pm standard deviations of assays performed in triplicate. K. pneumoniae ATCC 700606 and NTUH-K2044 were used as negative and positive controls, respectively. The optical density cut-off (ODc) value was determined using a previously described formula [42]: ODc $=$ average OD of the negative control $+(3 \times$ standard deviation of the negative control). Then, the ability of the isolate to produce biofilm was categorized as: strong (OD $>4 \times$ ODc); medium $(4 \times \mathrm{ODc} \geq \mathrm{OD}>2 \times \mathrm{ODc})$; weak $(2 \times \mathrm{ODc} \geq \mathrm{OD}>\mathrm{ODc})$; or none $(\mathrm{OD} \leq \mathrm{ODc})$.

\section{Galleria mellonella infection model}

The virulence of NYTJ35 was evaluated using the G. mellonella larvae infection model (Tianjin Huiyude Biotech Company, Tianjin, China) [2]. An overnight culture of $K$. pneumoniae was adjusted to $1 \times 10^{8} \mathrm{CFU} / \mathrm{mL}$ using phosphate-buffered saline. Then, G. mellonella larvae were injected with $10 \mu \mathrm{L}$ of the culture and incubated in the dark at $37^{\circ} \mathrm{C}$ for 5 days, with continuous monitoring of survival. K. pneumoniae NTUH-K2044 and K. pneumoniae ATCC 700603 were used as high-virulence and lowvirulence controls, respectively. All experiments were performed in triplicate.

\section{Whole-genome sequencing (WGS)}

WGS was used to identify resistance genes and virulence factors in the hvKP isolate. Approximately $10 \mu \mathrm{g}$ of DNA was extracted using the DNeasy UltraClean Microbial Kit (QIAGEN, Hilden, Germany) to establish two Illumina paired-end libraries with 500 and 2000 base pairs (average insertion lengths). Reads with any of the following characteristics were excluded from the raw data: $(i)$ undefined bases of $5 \mathrm{bp}$, (ii) low-quality $(\leq \mathrm{Q} 20)$ bases of $20 \mathrm{bp}$, (iii) contamination of adapter, or (iv) duplicates. SOAPdenovo version 1.05 was used to assemble the final cleaned reads, and the genome coverage for each strain was approximately $100 \times$. The CGE server (https:// cge.cbs.dtu.dk) was used to identify resistance genes. A phylogenic tree was constructed using the BacWGSTdb server with core genome multilocus sequence typing (cgMLST) [43]. The Oxford scheme was used for multilocus sequence typing (MLST), and the sequence types (STs) were determined using the MLST database (https:// bigsdb.pasteur.fr/klebsiella/klebsiella.html). 


\begin{abstract}
Abbreviations
ST: Sequence type; hvKP: Hypervirulent K. pneumoniae; KP: K. pneumoniae; ESBL: Extended spectrum beta-lactamase; MDR: Multidrug resistant; CKP: Classical K. pneumoniae; MALDI-TOF MS: Matrix-assisted laser desorption/ionization time-of-flight mass spectrometry; CFU: Colony forming units; MIC: Minimum inhibitory concentrations; CAZ: Ceftazidime; FEP: Cefepime; IPM: Imipenem; MEM: Meropenem; CZA: Ceftazidime/avibactam; ATM: Aztreonam; TZP: Piperacillin/tazobactam; NIT: Nitrofurantoin; AMK: Amikacin; LVX: Levofloxacin; TGC: Tigecycline; PCR: Polymerase chain reaction; OD: Optical density; cgMLST: Core genome multilocus sequence typing; MLST: Multilocus sequence typing; SNP: Single nucleotide polymorphism; WGS: Whole-genome sequencing; CLSI: Clinical and Laboratory Standards Institute; PAP: Population analysis profile; HCCA: A a-cyano-4-hydroxy-cinnamic acid; ASB: Asymptomatic bacteriuria; UTI: Urinary tract infection.
\end{abstract}

\section{Acknowledgments}

We would like to express our gratitude to the staff of the Department of Clinical Laboratory at Xiangya Hospital of Central South University for their assistance in collecting and identifying the K. pneumoniae isolates.

\section{Authors' contributions}

Study design: JL and MZ. Study conduct: FX, CM, and MT. Data collection: MT, FX, and CM. Data analysis: JL and MZ. Data interpretation: YH and HW. Drafting the manuscript: JL. Revising the manuscript content: MZ. Approving the final version of the manuscript: $J L$ and MZ. The final manuscript was read and approved by all authors.

\section{Funding}

The National Natural Science Foundation of China (No. 81702068), Natural Science Foundation of Hunan Province (No. 2020JJ4886), the Science Foundation of Hunan Health Commission in Hunan province (No. 202111000066), and college students' innovations special project (No. S2021105330304) supported this study.

\section{Availability of data and materials}

The datasets generated and analyzed during the current study are available in the NCBI BioProject repository, BioProject accession number: PRJNA753708.

\section{Declarations}

\section{Ethics approval and consent to participate}

This research was conducted using a protocol approved by the Central South University Ethics Committee (Hunan Province, China; ID 201703302) and according to the principles of the Declaration of Helsinki. Written informed consent was provided by the patient prior to the study.

\section{Consent for publication}

Not applicable.

\section{Competing interests}

There are no conflicts of interest declared by any of the authors.

\section{Author details}

'Department of Clinical Laboratory, Xiangya Hospital, Central South University, Changsha 410008, Hunan, China. ${ }^{2}$ National Clinical Research Center for Geriatric Disorders, Xiangya Hospital, Central South University, Changsha 410008, Hunan, China.

Received: 28 October 2021 Accepted: 31 January 2022

Published online: 07 February 2022

\section{References}

1. Rice LB. Federal funding for the study of antimicrobial resistance in nosocomial pathogens: no ESKAPE. J Infect Dis. 2008;197:1079-81.

2. Gu D, Dong N, Zheng Z, Lin D, Huang M, Wang L, et al. A fatal outbreak of ST11 carbapenem-resistant hypervirulent Klebsiella pneumoniae in a Chinese hospital: a molecular epidemiological study. Lancet Infect Dis. 2018;18:37-46.
3. Liu YC, Cheng DL, Lin CL. Klebsiella pneumoniae liver abscess associated with septic endophthalmitis. Arch Intern Med. 1986;146:1913-6.

4. Surgers L, Boyd A, Girard PM, Arlet G, Decré D. ESBL-producing strain of hypervirulent Klebsiella pneumoniae K2, France. Emerg Infect Dis. 2016;22:1687-8.

5. Wei DD, Wan LG, Deng Q, Liu Y. Emergence of KPC-producing Klebsiella pneumoniae hypervirulent clone of capsular serotype K1 that belongs to sequence type 11 in mainland China. Diagn Microbiol Infect Dis. 2016:85:192-4.

6. Li J, Huang ZY, Yu T, Tao XY, Hu YM, Wang HC, et al. Isolation and characterization of a sequence type 25 carbapenem-resistant hypervirulent Klebsiella pneumoniae from the mid-south region of China. BMC Microbiol. 2019;19:219.

7. Hosoda T, Harada S, Okamoto K, Ishino S, Kaneko M, Suzuki M, et al. COVID-19 and fatal Sepsis caused by hypervirulent Klebsiella pneumoniae, Japan, 2020. Emerg Infect Dis. 2021;27:556-9.

8. Pichler C, Büchsel M, Rossen JW, Vavra M, Reuter S, Kern WV, et al. First report of invasive liver abscess syndrome with endophthalmitis caused by a K2 serotype ST2398 hypervirulent Klebsiella pneumoniae in Germany, 2016. New Microbes New Infect. 2017:17:77-80.

9. Karlsson M, Stanton RA, Ansari U, McAllister G, Chan MY, Sula E, et al. Identification of a carbapenemase-producing hypervirulent Klebsiella pneumoniae isolate in the United States. Antimicrob Agents Chemother. 2019;63(7):e00519-19.

10. Guerra JM, Fernandes N, Morales Dos Santos AL, Barrel JSP, Petri BSS, Milanelo L, et al. Hypervirulent Klebsiella pneumoniae as unexpected cause of fatal outbreak in captive marmosets, Brazil. Emerg Infect Dis. 2020;26:3039-43.

11. Catalán-Nájera JC, Barrios-Camacho H, Duran-Bedolla J, Sagal-Prado A, Hernández-Castro R, García-Méndez J, et al. Molecular characterization and pathogenicity determination of hypervirulent Klebsiella pneumoniae clinical isolates serotype K2 in Mexico. Diagn Microbiol Infect Dis. 2019;94(3):316-9.

12. Marr CM, Russo TA. Hypervirulent Klebsiella pneumoniae: a new public health threat. Expert Rev Anti-Infect Ther. 2019;17:71-3.

13. Lu Y, Feng Y, McNally A, Zong Z. Occurrence of colistin-resistant hypervirulent Klebsiella variicola. J Antimicrob Chemother. 2018;73:3001-4.

14. Lu Y, Feng Y, McNally A, Zong Z. The occurence of colistin-resistant hypervirulent Klebsiella pneumoniae in China. Front Microbiol. 2018;9:2568.

15. Band VI, Weiss DS. Heteroresistance to beta-lactam antibiotics may often be a stage in the progression to antibiotic resistance. PLoS Biol. 2021;19:e3001346.

16. Wozniak JE, Band VI, Conley AB, Rishishwar L, Burd EM, Satola SW, et al. A Nationwide screen of carbapenem-resistant Klebsiella pneumoniae reveals an isolate with enhanced virulence and clinically undetected colistin heteroresistance. Antimicrob Agents Chemother. 2019;63(5):e00107-19.

17. Nicolle LE, Gupta K, Bradley SF, Colgan R, DeMuri GP, Drekonja D, et al. Clinical practice guideline for the Management of Asymptomatic Bacteriuria: 2019 update by the Infectious Diseases Society of America. Clin Infect Dis. 2019;68:1611-5.

18. Gu DX, Huang YL, Ma JH, Zhou HW, Fang Y, Cai JC, et al. Detection of colistin resistance gene $\mathrm{mcr}-1$ in hypervirulent Klebsiella pneumoniae and Escherichia coli isolates from an infant with diarrhea in China. Antimicrob Agents Chemother. 2016:60:5099-100.

19. Huang YH, Chou SH, Liang SW, Ni CE, Lin YT, Huang YW, et al. Emergence of an XDR and carbapenemase-producing hypervirulent Klebsiella pneumoniae strain in Taiwan. J Antimicrob Chemother. 2018;73:2039-46.

20. Bardet L, Baron S, Leangapichart T, Okdah L, Diene SM, Rolain JM. Deciphering heteroresistance to colistin in a Klebsiella pneumoniae isolate from Marseille, France. Antimicrob Agents Chemother. 2017;61(6):e00356-17.

21. Halaby T, Kucukkose E, Janssen AB, Rogers MR, Doorduijn DJ, van der Zanden AG, et al. Genomic characterization of colistin heteroresistance in Klebsiella pneumoniae during a nosocomial outbreak. Antimicrob Agents Chemother. 2016;60:6837-43.

22. Band VI, Satola SW, Smith RD, Hufnagel DA, Bower C, Conley $A B$, et al. Colistin heteroresistance is largely undetected among carbapenem-resistant Enterobacterales in the United States. mBio. 2021;12(1):e02881-20

23. Pomakova DK, Hsiao CB, Beanan JM, Olson R, MacDonald U, Keynan Y, et al. Clinical and phenotypic differences between classic and hypervirulent Klebsiella pneumonia: an emerging and under-recognized pathogenic variant. Eur J Clin Microbiol Infect Dis. 2012;31:981-9. 
24. Catalán-Nájera JC, Garza-Ramos U, Barrios-Camacho H. Hypervirulence and hypermucoviscosity: two different but complementary Klebsiella spp. phenotypes? Virulence. 2017;8:1111-23.

25. Russo TA, Olson R, Fang CT, Stoesser N, Miller M, MacDonald U, et al. Identification of biomarkers for differentiation of hypervirulent Klebsiella pneumoniae from classical K. pneumoniae. J Clin Microbiol. 2018;56(9):e00776-18.

26. Lin TL, Lee CZ, Hsieh PF, Tsai SF, Wang JT. Characterization of integrative and conjugative element ICEKp1-associated genomic heterogeneity in a Klebsiella pneumoniae strain isolated from a primary liver abscess. J Bacteriol. 2008;190:515-26.

27. Cheong HS, Kim SY, Wi YM, Peck KR, Ko KS. Colistin heteroresistance in Klebsiella pneumoniae isolates and diverse mutations of PmrAB and PhoPQ in resistant subpopulations. J Clin Med. 2019;8(9):1444.

28. Liu YY, Wang Y, Walsh TR, Yi LX, Zhang R, Spencer J, et al. Emergence of plasmid-mediated colistin resistance mechanism MCR-1 in animals and human beings in China: a microbiological and molecular biological study. Lancet Infect Dis. 2016;16:161-8.

29. Choi MJ, Ko KS. Loss of hypermucoviscosity and increased fitness cost in colistin-resistant Klebsiella pneumoniae sequence type 23 strains. Antimicrob Agents Chemother. 2015;59:6763-73.

30. Xu Q, Yang X, Chan EWC, Chen S. The hypermucoviscosity of hypervirulent K. pneumoniae confers the ability to evade neutrophil-mediated phagocytosis. Virulence. 2021;12:2050-9.

31. Ernst CM, Braxton JR, Rodriguez-Osorio CA, Zagieboylo AP, Li L, Pironti A et al. Adaptive evolution of virulence and persistence in carbapenemresistant Klebsiella pneumoniae. Nat Med. 2020;26:705-11.

32. Zhu J, Wang T, Chen L, Du H. Virulence factors in hypervirulent Klebsiella pneumoniae. Front Microbiol. 2021;12:642484.

33. Siu LK, Fung CP, Chang FY, Lee N, Yeh KM, Koh TH, et al. Molecular typing and virulence analysis of serotype K1 Klebsiella pneumoniae strains isolated from liver abscess patients and stool samples from noninfectious subjects in Hong Kong, Singapore, and Taiwan. J Clin Microbiol. 2011;49:3761-5.

34. Lin JC, Koh TH, Lee N, Fung CP, Chang FY, Tsai YK, et al. Genotypes and virulence in serotype K2 Klebsiella pneumoniae from liver abscess and non-infectious carriers in Hong Kong, Singapore and Taiwan. Gut Pathog. 2014;6:21.

35. Qu TT, Zhou JC, Jiang Y, Shi KR, Li B, Shen P, et al. Clinical and microbiological characteristics of Klebsiella pneumoniae liver abscess in East China. BMC Infect Dis. 2015;15:161.

36. Guo Y, Wang S, Zhan L, Jin Y, Duan J, Hao Z, et al. Microbiological and clinical characteristics of hypermucoviscous Klebsiella pneumoniae isolates associated with invasive infections in China. Front Cell Infect Microbiol. 2017;7:24.

37. Clinical and laboratory standards institute (CLSI): The performance stands for antimicrobial susceptibility testing. 2020 The M100-S30

38. Sherman EX, Wozniak JE, Weiss DS. Methods to evaluate colistin heteroresistance in Acinetobacter baumannii. Methods Mol Biol. 2019;1946:39-50.

39. Li J, Zou M, Dou Q, Hu Y, Wang H, Yan Q, et al. Characterization of clinical extensively drug-resistant Pseudomonas aeruginosa in the Hunan province of China. Ann Clin Microbiol Antimicrob. 2016;15:35.

40. Li G, Shi J, Zhao Y, Xie Y, Tang Y, Jiang X, et al. Identification of hypervirulent Klebsiella pneumoniae isolates using the string test in combination with galleria mellonella infectivity. Eur J Clin Microbiol Infect Dis. 2020;39:1673-9.

41. Coffey BM, Anderson GG. Biofilm formation in the 96-well microtiter plate. Methods Mol Biol. 2014;1149:631-41.

42. Devanga Ragupathi NK, Muthuirulandi Sethuvel DP, Triplicane Dwarakanathan H, Murugan D, Umashankar Y, Monk PN, et al. The influence of biofilms on carbapenem susceptibility and patient outcome in device associated K. pneumoniae infections: insights into phenotype vs genomewide analysis and correlation. Front Microbiol. 2020;1 1:591679.

43. Ruan Z, Feng Y. BacWGSTdb, a database for genotyping and source tracking bacterial pathogens. Nucleic Acids Res. 2016;44:D682-7.

\section{Publisher's Note}

Springer Nature remains neutral with regard to jurisdictional claims in published maps and institutional affiliations.

Ready to submit your research? Choose BMC and benefit from:

- fast, convenient online submission

- thorough peer review by experienced researchers in your field

- rapid publication on acceptance

- support for research data, including large and complex data types

- gold Open Access which fosters wider collaboration and increased citations

- maximum visibility for your research: over $100 \mathrm{M}$ website views per year

At BMC, research is always in progress.

Learn more biomedcentral.com/submissions 